\title{
Development of Zigbee-Wifi-Wimax Hybrid Wireless Sensor Network based Telemedicine System
}

\author{
Shahida Khatoon \\ Dept. of Electrical Engineering \\ Faculty of Engineering \& Tech. \\ Jamia Millia Islamia, New Delhi
}

\author{
Manoj Kumar Singh \\ Dept. of Electrical Engineering \\ Faculty of Engineering \& Tech. \\ Jamia Millia Islamia, New Delhi
}

\author{
Ahmad Saad Khan \\ Dept. of Electronics \& Comm. \\ Faculty of Engineering \& Tech. \\ Jamia Millia Islamia, New Delhi
}

\begin{abstract}
This paper is an outcome of an on-going research project sponsored by department of (DST), Ministry of Science and Technology, Government of India, in which a wireless sensor network based on ZigBee-WiFi-Wi-Max hybrid wireless sensor network (WSN) has been developed. This system has been used for implementing telemedicine system as an information gateway between old age homes and nearby hospitals, for the purpose of transmitting health data wirelessly over a distance of about $20 \mathrm{~km}$ range. This system is developed in two separate modules, one consisting of ZigBee based WSN which is applied for wireless data transmission up to a distance of two kilometres, and the other comprises of WiFi-Wi-Max based WSN used to transmit data up to a distance of twenty kilometres. The hybrid wireless network has been tested for distance range, speed of data transfer and time delays for bulk data transfer. Several observations were collected and it has been proved experimentally that the developed system is low cost, consumes relatively low power, and transmits data efficiently without significant time delay in bulk data transmission. Another solution which has been designed and validated is to transfer all data to a database residing on web. This database can be accessed via 3G - GRPS connection on PDA, Mobile phones at any location across the globe. For areas without pre-existing physical cable or landline telephone networks, wireless application protocol (WAP) may be a viable alternative for broadband access between remote ZigBee networks and hospital information centre that has been economically unavailable. In the ZigBee-Wi-Max hybrid system first low distance information (within old age home) is transmitted wirelessly using ZigBee technology. Then this information is transferred to Web server and it can be assessed on Laptops/ Computers having internet connection, mobile phones, personnel digital assistant (PDAs) using GPRS connection. The performance of the hybrid system has been evaluated based on numeric result of testing.
\end{abstract}

\section{Keywords}

ZigBee, Wi-fi, Wi-Max, Android App, GRPS, PDA, WAP, Pulse Oxi meter, wireless application protocol (WAP), personnel digital assistant (PDA)

\section{INTRODUCTION}

The problem of safety and medical health condition of the residents are major causes of concern in the old age homes. It is not very uncommon that we hear that an elderly person got robbed, injured, or even brutally murdered in his own home by some stranger or by domestic help. The elderly having sudden high/low blood pressure, being fallen, having asthma attack or having cardiac arrest when he/she is alone in his home or in an old-age home is also very frequently occurring phenomenon. To make sure the medical well being of the elderly person and to cater to his safety issue, it is highly recommended that a continuous monitoring system should be fitted in the old age homes. Due to our urban lifestyle and socio-economic status, these days it is not possible for the grown up children to provide vigilance to their parents for twenty four hours, they need to be away from home for their jobs and business. Moreover domestic help and other careproviders are also proving to be dangerous, since most of the time the elderly person is alone in their homes. These constraints make the elderly physically vulnerable group to become soft targets. Thus continuous health and fitness monitoring, security vigilance and safety surveillance is utmost necessity in any old age home.

In present research wireless sensor technology is used to show that the health data can be transmitted from the patient room to a server room, without having additional cables to run through the two destinations. The developed system is able to store medical records of elderly and transmits the data wirelessly over a distant hospital. The developed system is helpful in connecting rural dispensaries to nearby urban hospitals wirelessly. The observations taken from experimental set up have demonstrated that signals can be transmitted successfully on the whole floor from upstairs to downstairs with good quality of communication and a penetrating ability of signals under $2.4 \mathrm{GHz}$ is higher than that available in other bands. Monitor nodes can easily be added or removed in the system, it is also convenient to expand the network. As an expansion of existing wired health monitoring systems it can enhance the flexibility of information collecting, while reducing the cost of communication network in residential buildings, thus improving the applied value of health monitoring and practical value of control information system.

The medical data of each occupant of the old age home is transmitted through ZigBee network and monitored by medical staff through wired ward desktop. So doctor or nurse can observe the real-time medical data/waveforms. Besides, patients' EGC abnormality can also be detected and informed so that medical staffs can handle the emergency issue as soon as possible. If the medical staffs desire more mobility within the building, they can also carry ZigBee-enabled PDA with which they can receive patients' health information. In this system, mesh network is employed and information flows from ECG to ZigBee coordinator (ZC) through single or multiple ZigBee Routers (ZR). As ZigBee covers maximum distance of 700 meters it has been made to interface with WiMax module to transmit the medical records to a nearby health service provider. This ZigBee-Wi-Max hybrid wireless network has been tested successfully over a distance of $2 \mathrm{~km}$. If the Wi-Max dongles can be mounted on a high rise tower such as given by telephone line providers, it can transmit the data over a distance of $20 \mathrm{Km}$. 


\section{LITERATURE SURVEY}

Many patient remote monitoring devices were reported in the literature [1-9]. An otolaryngology procedure was reported in [1]. The unit helps physician to perform a variety of procedures such as nasal endoscopy, biopsies and removal of foreign bodies. The developed protocol is implemented and tested to monitor the medical condition of a large number of patients [2]. The protocol receives the temperature and pressure of a patient using a mobile device that is attached to the patient's body via short messaging system (SMS) message. The mobile device does not have data logging capabilities, nor does it have download and diagnosis features. The clinical usefulness of a wireless personal digital assistant (PDA) based on a GPRS-capable cellular phone and an Internet application for remote monitoring of real-time vital signs was discussed in [3]. A wireless PDA-based physiological monitoring system for patient transport that uses wireless local area network (WLAN) technology to transmit patient's bio-signals in real-time to a remote central management unit was presented in [4]. Most of the above systems do not have logging capabilities to allow the patients to be away for a week or so without communicating with their physician. Instead, biomedical signals are transmitted in realtime to the physician in these solutions. This paper discusses the design of wireless mobile logging device using an embedded system platform. The unit is used to measure a patient's blood glucose concentration in units of milligrams per deciliter $(\mathrm{mg} / \mathrm{dL})$, heart rate in beats per minute $(\mathrm{bpm})$, and pulse oximetry in percentage of concentrated blood oxygen (\% SpO2). The architecture of the patient monitoring systems is shown in Figure 1. While the patient practicing his/her normal day activities, the acquired signals values are logged for a period of time or until the next physician visit. The platform stores a set of $\mathrm{x}$ number of values for each signal. The data of a trial period of one week is downloaded to a PC using the RS-232 port and analyzed using a custom-built software application. Rapid prototyping options and ease of firmware upgrade are crucial for such a device. For these reasons a Matlab program was developed and customized for low-power consumption and reduced measurement time. The system architecture is given in section II, whereas section III shows the embedded-system hardware design methodology. A prototype for the system was successfully built and tested as explained in section IV.

\section{METHODOLOGY}

The prototype developed has been divided into five units.

3.1 Unit 1 consists of wearable medical sensors used to health information of the elderly on regular basis. The vital signs of the patient are taken by common medical sensors such as temperature sensor, heart beat sensor, pulse oximeter, and motion detector. These sensors are attached to the microcontroller board so that the health data can be processed by the interfaced computer system and can be transmitted to the wireless sensor network formed by ZigBee trans-receiver module.

\subsubsection{Pulse Rate Sensor}

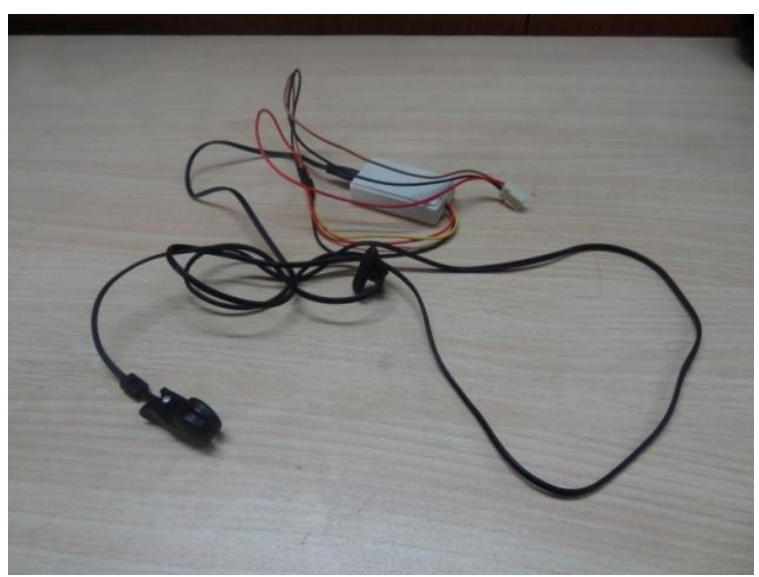

Fig. 1: Grove - Ear-clip Heart Rate Sensor

A patient's heart beat count is the first method of diagnosis practiced by almost all doctors .In our model we have used Grove ear clip heart rate sensor consisting of an ear clip connected to patient's earlobe and a receiver module. The heart rate is displayed on a screen via the serial port and can be saved for analysis. This particular sensor is used because it is light weighed, lesser wattage rating, and has high accuracy of measurements.

\subsubsection{Temperature sensor}

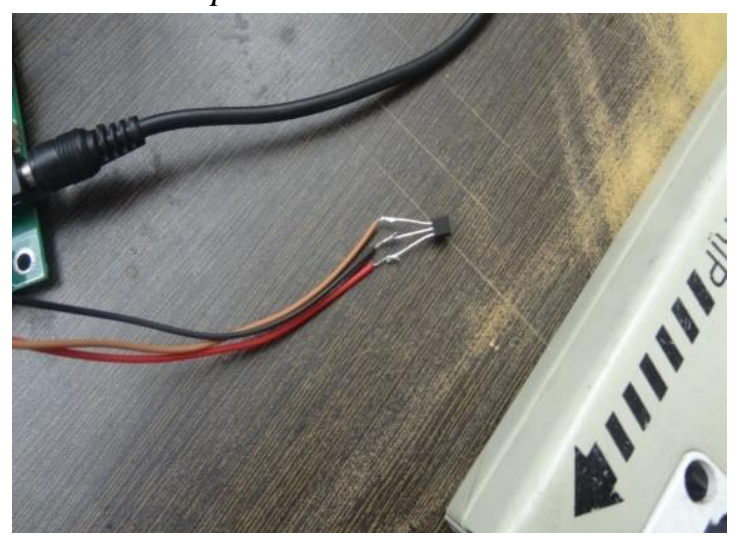

Fig.2: Temperature sensor

The temperature sensors used for recording patients body temperature is of LM 35 series which is rated to operate over $\mathrm{a}-55^{\circ}$ to $+150^{\circ} \mathrm{C}$ temperature range it draws only $60 \mu \mathrm{A}$ from its supply, it has very low self-heating, less than $0.1^{\circ} \mathrm{C}$ in still air . It can be used with single power supplies, or with plus and minus supplies The advantages of the sensor are its high sensitivity, low current consumption, low output impedance, precise calibration, and ease of operation and control. 


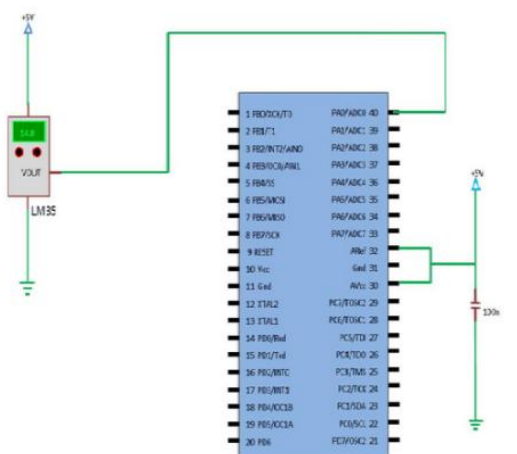

Fig. 3: Interfacing LM 35 with Atmega 16 micro controller

\subsubsection{Pulse Oximeter}

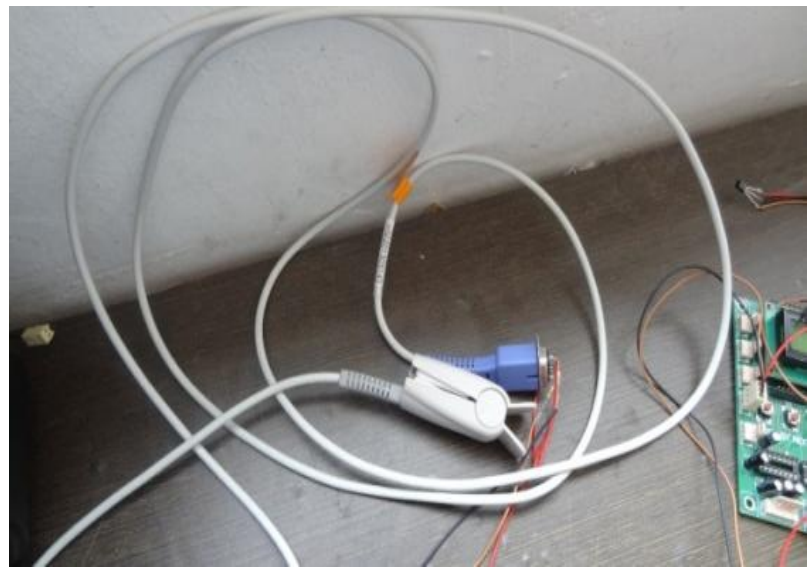

Fig. 4: Pulse Oximeter

The saturation of patients' blood haemoglobin level is monitored using pulse oximeter. It is clamped between the patient's fingertips and light of two different wavelengths is allowed to pass through the patient's body to a photo detector. The changing absorbance at each of the wavelengths is measured, which determines the absorbance of blood haemoglobin. This type of sensor is used for very critical patients normally ICU patients.

\subsubsection{Motion Detector}

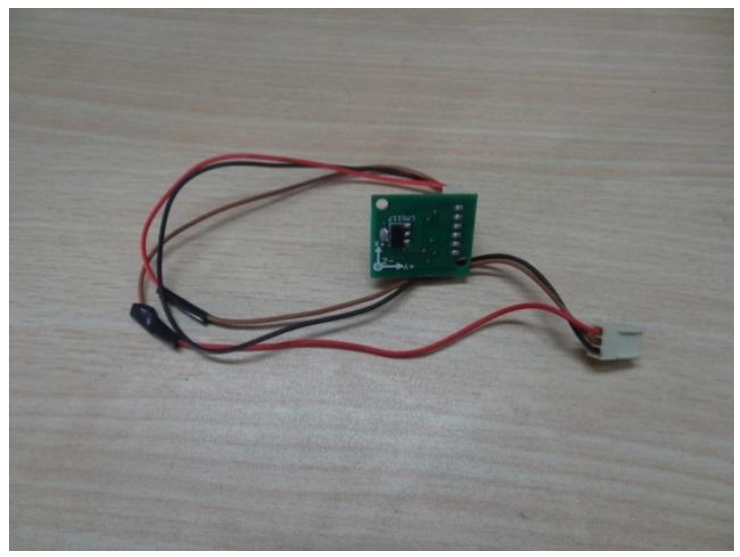

Fig. 5: Motion Detector

In order to keep an eye on the daily activities of an elderly in the house a motion detector is used. The accelerometer used for this purpose measures the vibration which develops a force which squeeze's a piezoelectric material used in the accelerometer. This produces an electrical charge that is proportional to the force exerted upon proportional to the acceleration. This sensor proves very useful to raise an alarm if an elderly gets unconscious or is fallen down. Therefore it is also sometime referred to as fall detector.

\subsubsection{Interfacing medical sensors with micro controller board}

A microcontroller is a small computer on a single integrated circuit containing a processor core, memory, and programmable input/output peripherals. A Microcontroller can be defined as a "Computer-on-Chip".Just as a Personal Computer has input devices like keyboards, mouse, etc. output devices like monitor, printer, etc and the Central Processing Unit. Microcontroller also has (a) Input/Output ports, (b) A Processor embedded into a single chip (C) Memory.

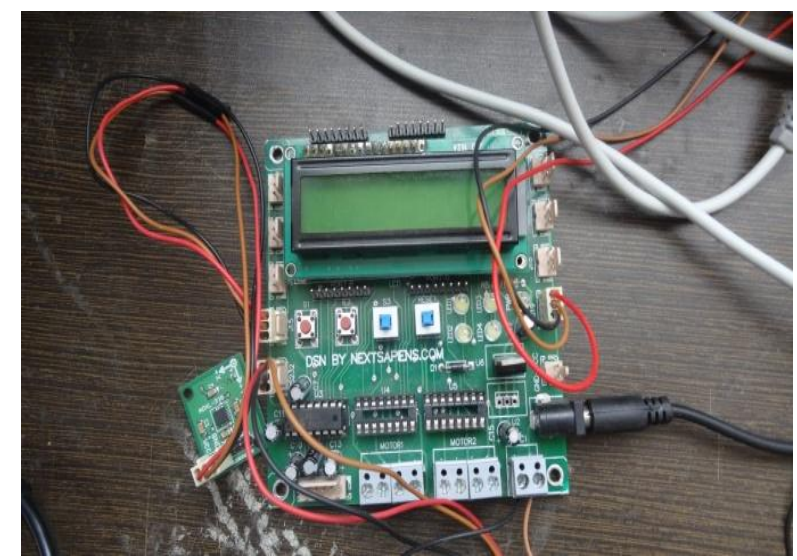

Fig. 6: Microcontroller prtoboard

The development board is a hand-held, battery powered microcontroller board. Based on the Atmel Atmega16 microcontroller, the development board includes $16 / 32 \mathrm{~K}$ of flash RAM, outputs for four motors or two stepper motors, inputs for a variety of sensors and a $16 * 2$ alphanumeric screen. The development board runs BASCOM-AVR,a cross platform, specialized AVR of the basic programming language.

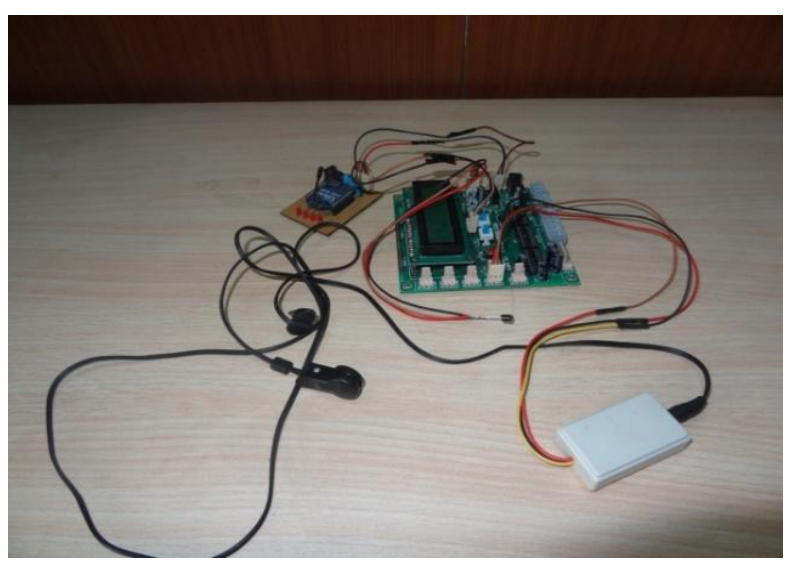

Fig. 7: Medical sensors interfaced with proto-board 


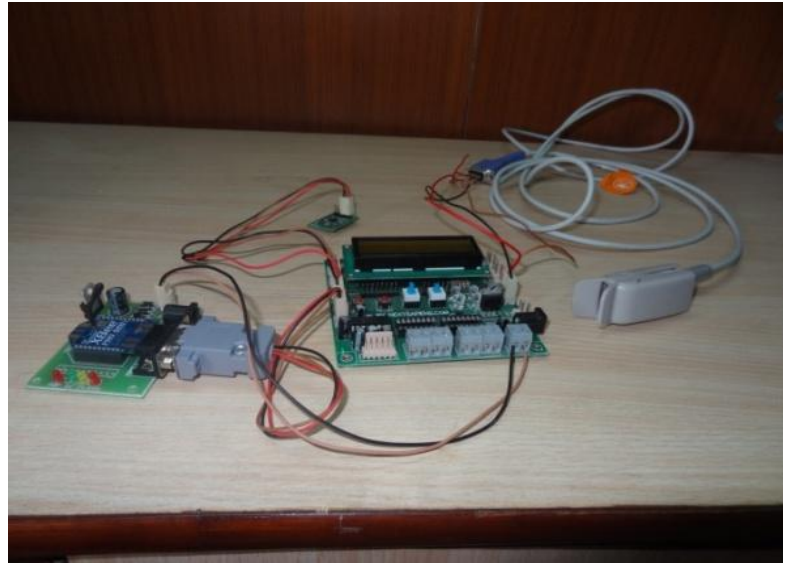

Fig. 8: ZigBee transmitter attached with proto board

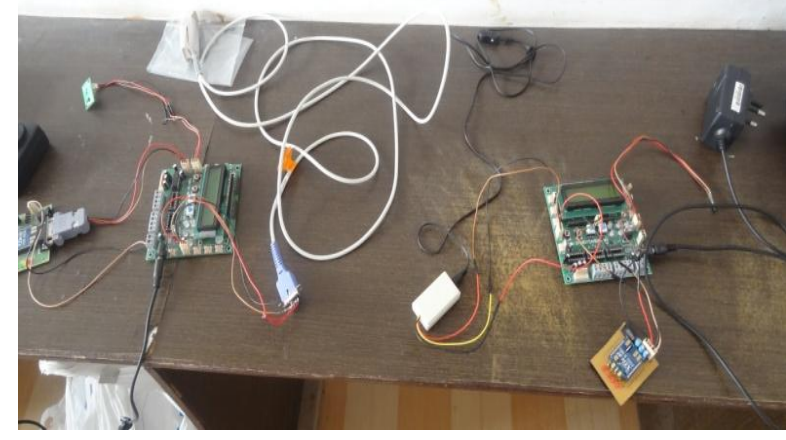

Fig. 9: Zigbee transmitter receiver attached with medical sensors

3.2 UNIT 2: This unit consists of a central data recording computer system. This system is attached with the ZigBee trans-receiver module. The main task of this unit is to receive the medical data from the unit 1 ( from the sensors via ZigBee module) and store the data received in the data base

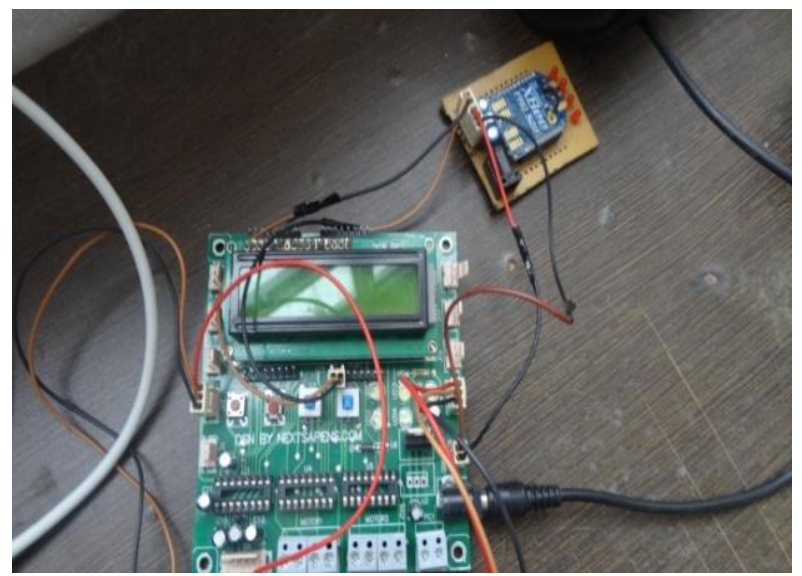

Fig. 10 : ZigBee reciever connected to $\mathrm{MC}$

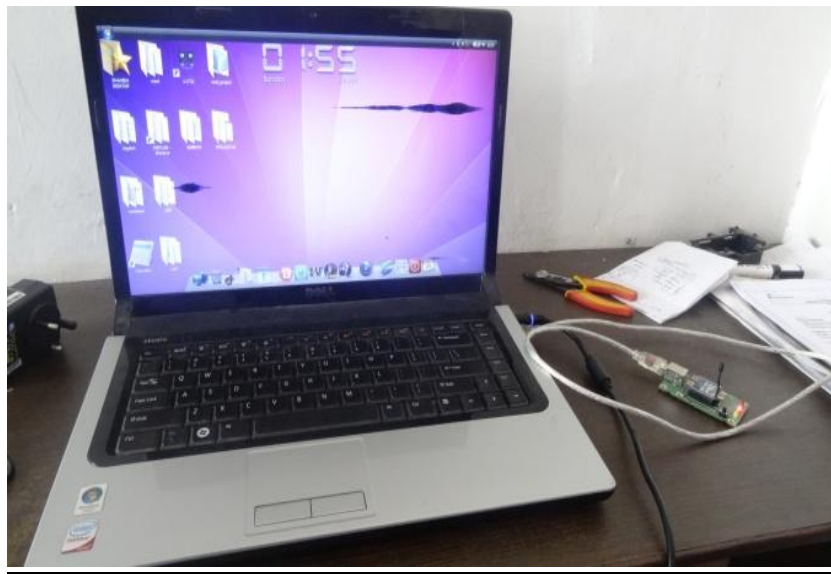

Fig. 11 : ZigBee receiver connected to laptop

\subsection{UNIT 3: Telemedicine Software}

This project presents a system that provides a continuous health monitoring service for people. Temperature , HB, Motion and pulse signals, are measured from the sensors and are processed by a built-in microcontroller. The processed data are then transmitted by Zigbee wireless transmission. Finally the received data is stored in the PC. In the PC a coding is written using MATLAB for transmitting and receiving the data and then storing it in the excel file. It can facilitate doctors in diagnosis and improve the efficiency and quality of medical administration.

The ATmega16 microcontroller is programmed to interfaced with pulse oximeter, LM35 Temperature sensor and to the SIM module to shoot the SMS in the case of any abnormlity in patient's health. This programming is done in BASCOM. BASCOM Compiler is used for programming the controller unit to which is also connected the SIM module.

MATLAB programming is done in computer to show the temperature in GUI window, to show the pulse rate in GUI window and to store the data in excel file.

When we push the pulse button the value read by pulse oximeter is received serially and similarly when we press the temp button the value of temperature sensor is received and stored in the excel sheet respectively. Pushing the save button we can store the values in the excel sheet.

\section{Front End Screens of Tele- Medicine Software developed}

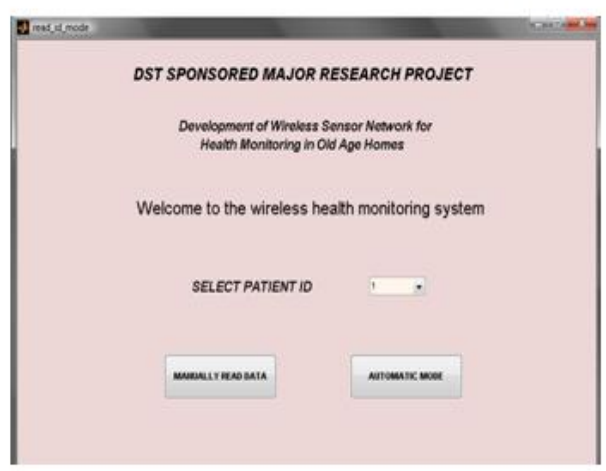

Fig.12: Telemedicine software front end 


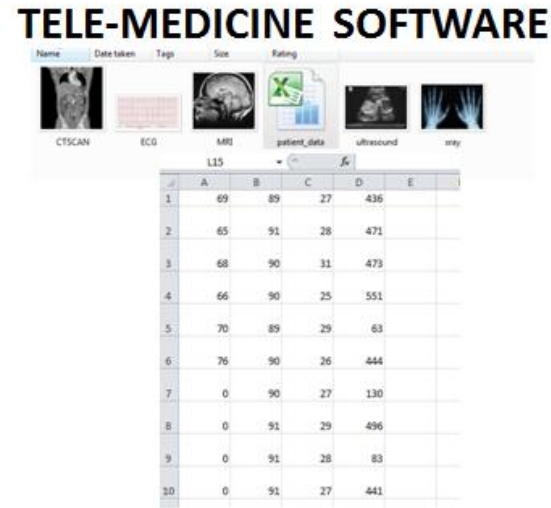

Fig. 13: Telemedicine software back end

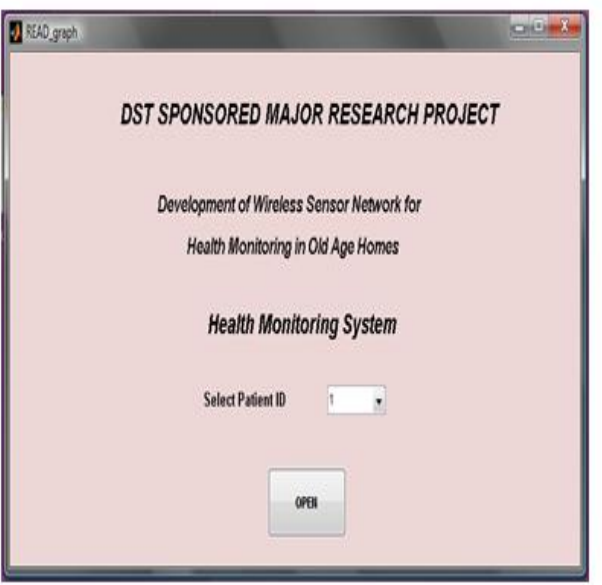

Fig. 14: Telemedicine GUI

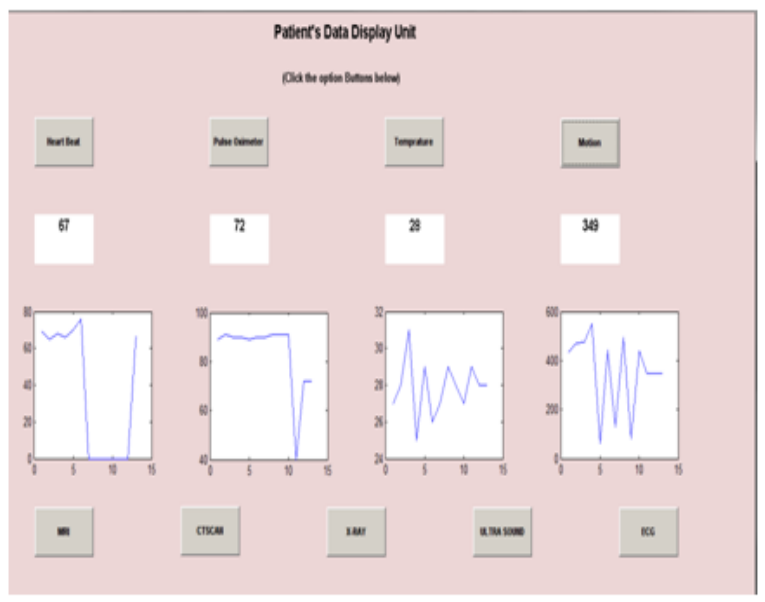

Fig. 15: Telemedicine GUI 2
3.4 UNIT 4: This unit is meant for transferring of data from Unit 2 (consisting of a computer) to another computer which is located at certain distance from it using wireless data transfer system. In designing the wireless data transfer system between unit 2 and 3, we have tested various existing technologies like Wifi, Wimax, Website and Mobile application and drawn a comparison between them in terms of range, speed of data transfer and their limitations.

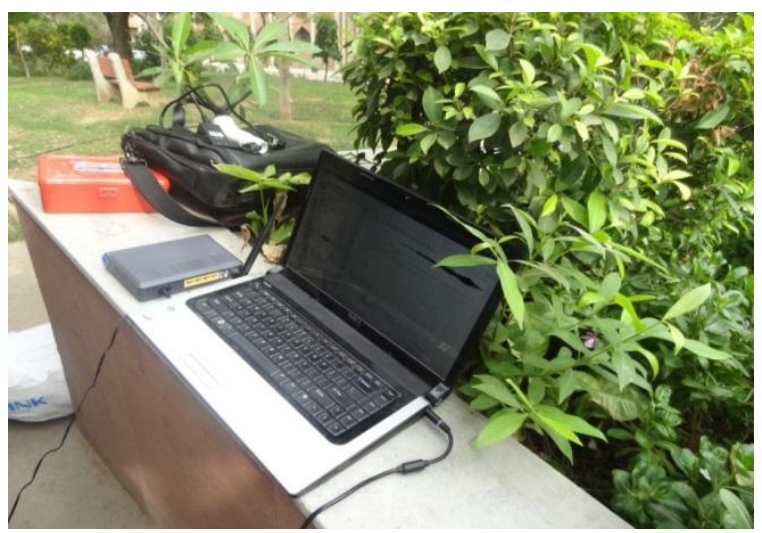

Fig.16 : Wi-Fi Modem

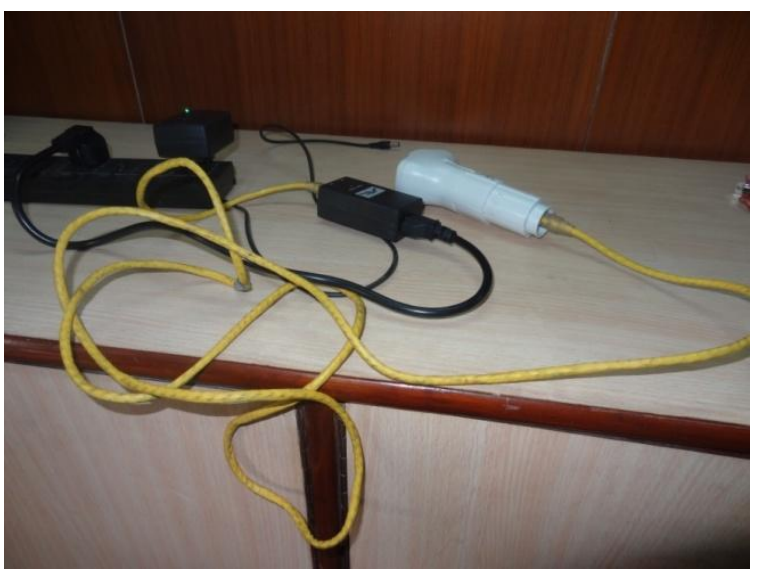

Fig.17: Wi-Max Dongle

3.5 UNIT 5: Tele Medicine web based application- We have developed a tele- medicine website software from where a user can transfer the data using it. It consists of:

Admin panel is used for uploading data to the data base. It is protected with the password. A user has to log in to the admin panel to upload the data files of the patient from the base station computer to server. On logging, user will select the patient id and will upload the data files for that patient. Also, via admin panel, user can create the login account of the doctors who can access the data.

Database: All the files reside at the server.

User front end: We have designed a simple front end for the doctors. They will be provided with a username and a password through which they can access the details of the patient. On logging, they will see the patients Id and on clicking patient id they can see the records of that particular patient. The design of front end is very simple and can also be access on the mobile phone via GPRS connection. The problem that can be faced in accessing it on mobile phone is of the resolution of the screen. Since the resolution of the screen of the mobile phones varies, the display of the website will not be uniform in that case. 


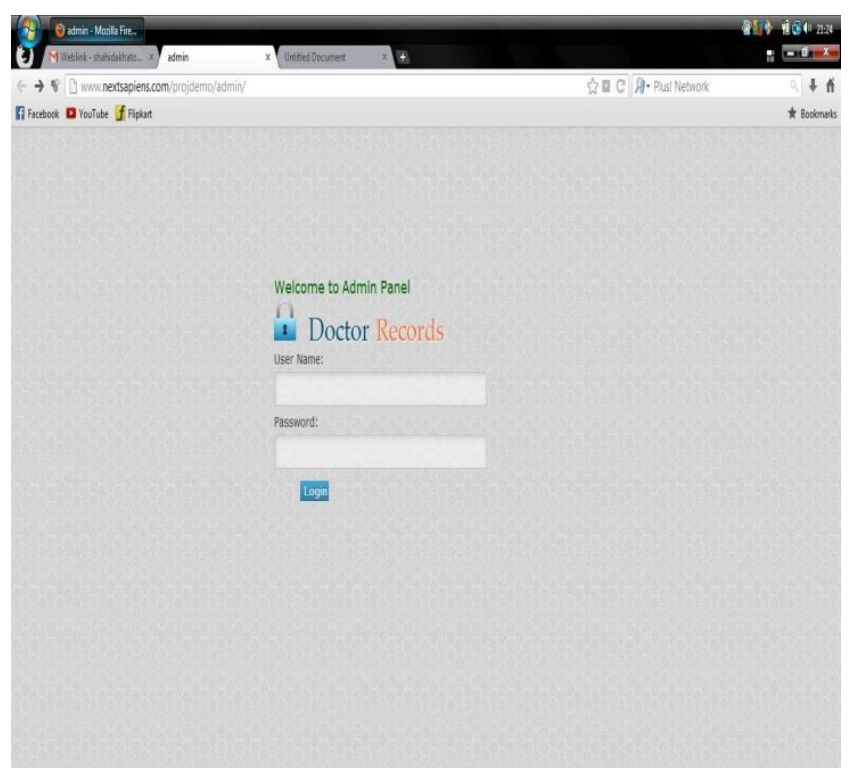

Fig.18: Internet based doctor's log-in web page

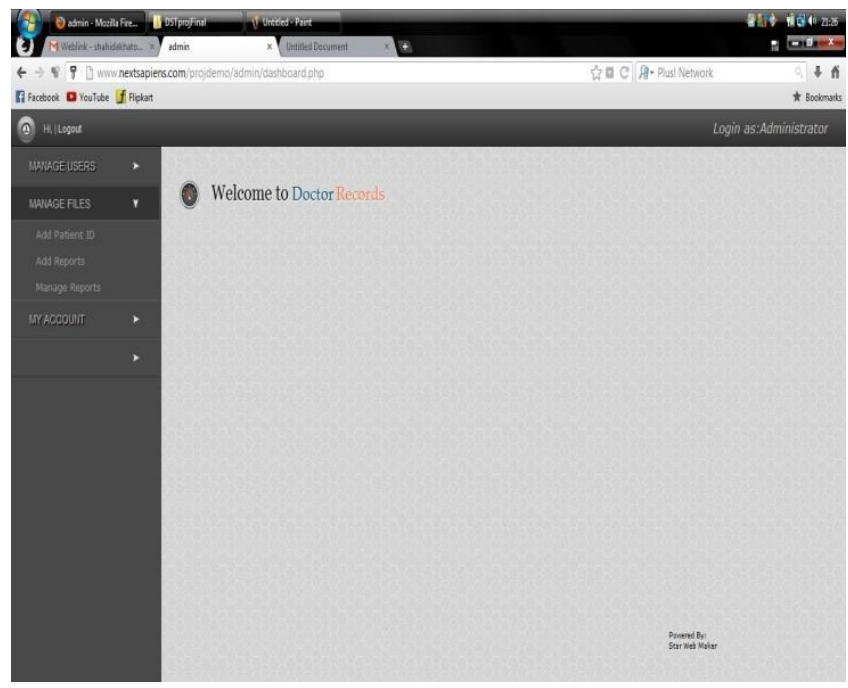

Fig. 19: Web based medical record page

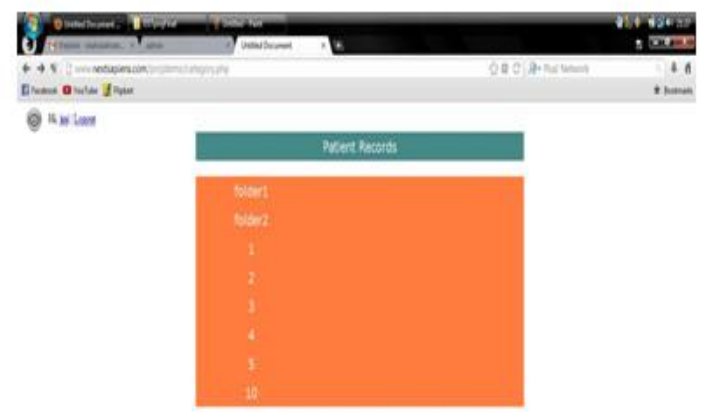

Fig. 20: Web Based App Front End

\subsection{UNIT 6: Wireless Communication with Android App}

Recently there has been an increase in mobile app development for various applications due to their portability advantage and ease of operation. An Android App has been developed for our tele-medicine system. This App will enable the medical experts to access the medical health data of their patients on their cell phones on the go, all over the world. This app will give the benefit of bringing many experts from across the globe together for a particular case as the medical data will be available on their mobile phones, so many medical practitioners may consult each other for any particular case.This app is also connected to the database mentioned above and will be accessed in the same manner as we are doing it in case of website. The doctor has to press "Launch Application" button. Then the next screen will pop-up with "Select patient-id" button. By selecting the id of the patient the patient's medical data like ECG, X-Ray, MRI, Ultra Sound, Heart Beat, temperature, oxygen level will be made available to the doctor on his mobile phone screen.

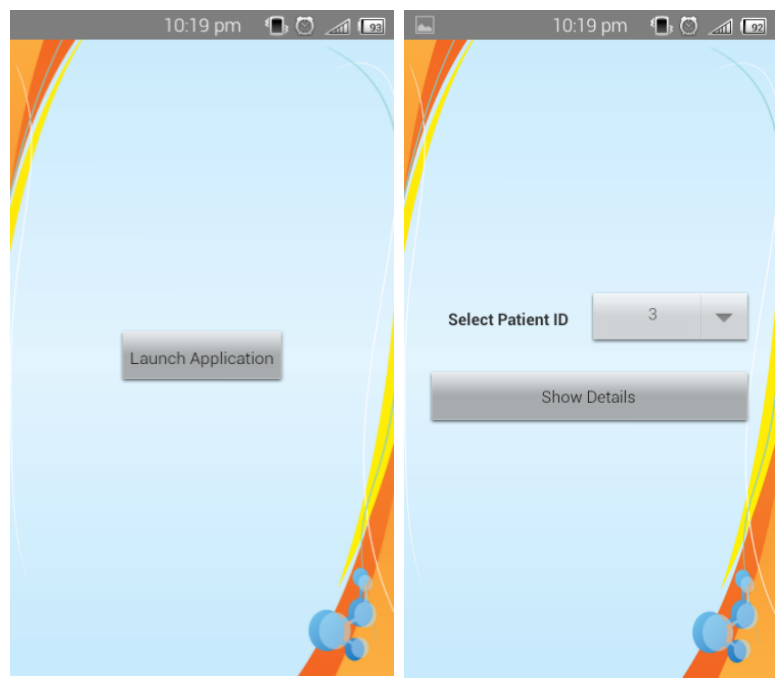

Fig. 21: Android App Snapshot

\section{CONCLUSION}

The developed system does the health monitoring task and transmits the data wirelessly over a range of two kilometres by means of ZigBee module. The ZigBee wireless sensor network have been successfully tested giving ATmega micro controller distant data monitoring and control. Experiments have proved that signals can be well transmitted in the whole floor from upstairs to downstairs with good communication quality. The penetrating ability of signals under $2.4 \mathrm{GHz}$ is higher than that in other bands. The distance range of the proposed ZigBee wireless sensor network is tested successfully for a distance utpo $700 \mathrm{~m}$. As the difficulty of clear LOS encountered in Wi-Max WSN it is tested with WiFi Modem. Wi-Fi gave a good signal strength for a distance of $1 \mathrm{~km}$ without LOS. The speed of data transmission was lesser as compared to Wi-Max Dongle. In order to overcome the problem of LOS of Wi-Max and distance range of ZigBee and $\mathrm{Wi}-\mathrm{Fi}$, an internet web page has been developed and tested successfully which will communicate data around the globe with very fast rate with or without clear LOS. An android Application has also been developed for transferring the data to Doctor's cell phone so that emergency situations can be connected to his mobile phone. The developed system has been tested in a nearby hospital. The prototype model was 
demonstrated to the doctors and they appreciated the efforts. With the use of wireless sensor technology, it has been shown that the health data can be transmitted from the patient room to a server room, without having to run additional cables through the two destinations. The developed circuit detects any intrusion and transmits the data wirelessly over a range of two kilometres by means of ZigBee module. The ZigBee wireless sensor network have been successfully tested giving ATmega micro controller distant data monitoring and control.

Experiments have proved that signals can be well transmitted in the whole floor from upstairs to downstairs with good communication quality. And penetrating ability of signals under $2.4 \mathrm{GHz}$ is higher than that in other bands. The distance range of the proposed ZigBee wireless sensor network is tested successfully for a distance upto $700 \mathrm{~m}$. The distance range can be extended using Wi_Max technology up to fifty kilometres. This ZigBee-Wi_Max hybrid wireless communication module has been tested successfully for a distance up to two km. As Wi-Max technology comes under point-to-point communication protocol, it can give a distance range up to fifty $\mathrm{km}$ if its antennas are mounted on a high rise tower with clear line of sight. The basic advantage of the developed WSN telemedicine system is that the monitor nodes can easily be added or removed in the system, it is also convenient to expand the network. As an expansion of existing wired security systems it can enhance the flexibility of information collecting, while reducing the cost of building safety system communication network in residential buildings. So it improves the applied value of safety monitoring and practical value of control information system. Further work on the system needs to be done to expand the scope of the system. This system is only a demonstrator of a single room communicating with a base-station, it can be expanded further to have multiple rooms communicating with a single base station.

\section{REFERENCES}

[1] F. Sigari, J. Schneider and J. M. Pinto, " Use of a mobile operative unit for in-patient otolaryngology procedures" Otolaryngology - Head and Neck Surgery, Volume 136, Issue 1, January 2007, Pages 125- 127

[2] A.R. Al-Ali, M. Al-Rousan and T. Ozkul, " Implementation of experimental communication protocol for health monitoring of patients", Computer Standards \& Interfaces, Volume 28, Issue 5, June 2006, Pages 523- 530
[3] P. Klootwijk, S. P. Nelwan, T. B. Van Dam and S. H. Meij, " Wireless (GPRS-Based) mobile real-time patient monitoring", Journal of the American College of Cardiology, Volume 41, Issue 6, Supplement 2, 19 March 2003, Page 528.

[4] Y. Lin, I-C. Jan, Ko, P.C.-I., Y. Yu Chen, J.-M. Wong and G.-W Jan; " A wireless PDA-based physiological monitoring system for patient transport", Information Technology in Biomedicine, IEEE Transactions on Volume 8, Issue 4, Dec. 2004 Page(s):439 - 447

[5] Texas Instruments, TLV2254I Advanced LinCMOSTM Rail-to-Rail Very Low-Power Operational Amplifiers, February 1997 revised March 2001.

[6] Smiths Medical, Inc., 31392B Micro power PCB Technical Description, November 2005.

[7] Barrett and D. Pack, Embedded Systems Design and Applications with the 68HC12 and HCS12. New Jersey: Prentice Hall, 2005.

[8] Smiths Medical, Inc., 31392B Micro power PCB Technical Description, November 2005.

[9] M. El Korek, R. Aloul, T. Landolsi, A.R. Al-Ali, and Y. Al-Assaf, "Portable Integrated Multi-Signal Patient Monitoring System," Proceedings of the IEEE conference on Innovation in IT, Dubai, 2006.

[10] A. P. Abidoye, N. A. Azeez, A. O. Adesina, K. K. Agbele, H. O. Nyongesa, "Using Wearable Sensors for Remote Healthcare Monitoring System," Journal of Sensor Technology, 2011, 1, 22-28.

[11] C. Rotariu, H. Costin and Gladiola Andruseac, et al., An Integrated System for Wireless Monitoring of Chronic Patients and Elderly People. 15th International Conference on System Theory, Control and Computing (ICSTCC).2011

[12] N.K. Suryadevara, M.T. Quazi and S.C. Mukhopadhyay, Intelligent Sensing Systems for measuring Wellness Indices of the Daily Activities for the Elderly. 8th International Conference on Intelligent Environments. 2012.

[13] CH. Wang, Q. Wang and SZ. Shi, A Distributed Wireless Body Area Network for Medical Supervision. IEEE International Conference on Instrumentation and Measurement Technology (12MTC). 2012. 